\title{
The impact of close binary evolution on the properties of the WR-bump emission lines of Wolf-Rayet galaxies
}

\author{
Joris Van Bever ${ }^{1}$ and Dany Vanbeveren ${ }^{1,2}$ \\ ${ }^{1}$ Astrofysisch Instituut, Vrije Universiteit Brussel, \\ Pleinlaan 2, B-1050 Brussel, België \\ ${ }^{1}$ Technische Hogeschool, Campus Vesalius, B-3000 Leuven, België
}

\begin{abstract}
We present the results of a study on the behaviour of the blue and red WR emission bumps (around $4650 \AA$ and $5808 \AA$ ) and of the nebular contribution to He II $\lambda 4686$ in evolving young starburst regions (such as Wolf-Rayet galaxies), containing a non-negligible binary population. Calculations were made for solar metallicity and $1 / 20$ solar. The population synthesis program uses an extended library of stellar evolutionary tracks of single stars and binaries, computed using the most recent stellar wind mass loss rates during RSG, LBV and WR stages. In the case of binaries, we account in detail for the effects of Roche lobe overflow, mass transfer and mass accretion, common envelope evolution, the spiral-in process, asymmetric kicks to neutron stars as a result of their supernova explosion, etc. This research is part of a more extensive project to explore every possible impact of massive binaries on stellar populations.
\end{abstract}

\section{Introduction}

The signatures of Wolf-Rayet stars in the spectra of unresolved starbursts are best seen in the optical. Among the brightest features are the complex of emission lines around $4650 \AA$ called the 'blue bump' (containing stellar $\mathrm{N} \mathrm{III} / \mathrm{N} \mathrm{V}$ $\lambda 4640$, stellar C III/C IV $\lambda 4650$ and stellar+nebular He II $\lambda 4686$ ), and the 'red bump' formed of the multiplet C IV $\lambda \lambda 5808-5812$. Using our extended library of binary evolutionary computations in combination with our binary population synthesis (PNS) code, we investigated the influence of binaries on the behaviour of the two WR-bumps in an evolving starburst with metallicity $Z=0.001$ or $Z=0.02$. The binary PNS code accounts for all binary evolutionary scenarios in a detailed way. We just mention two important issues. First, the importance of the adopted mass loss rate for WR stars. These were taken from Vanbeveren, van Rensbergen \& de Loore (1998): $\log \dot{M}_{\mathrm{WR}}=\log L-10$ at $Z=0.02$. At $Z=0.001$ we computed models using the same $\dot{M}$-law, as well as a scaled law according to $\sqrt{Z}$. Second, even if mass transfer is very non-conservative, a binary can still produce a rejuvenation effect. The large decrease in orbital period causes many binaries to merge. The end product is most likely a well mixed star, effectively less evolved than the originally most massive binary component. Therefore, binaries will always produce some rejuvenation, whether mass transfer is conservative or not. 


\section{The nebular He II $\lambda 4686$ emission line}

Nebular He II $\lambda 4686$ emission develops when very hot (several $10^{4} \mathrm{~K}$ ) and luminous stars produce significant continuum emission below $228 \AA$. The main contribution to this ionizing radiation comes from WR stars. Crucial to the behaviour of this ratio is the radius used to represent the core radius of the star as defined by Schmutz, Leitherer \& Gruenwald (1992), which is needed to assign the correct stellar continuum to a given evolutionary model. Usually, the hydrostatic radius of the star is used, but because of the uncertain structure in the subsonic region, we calculated models in which we set the Schmutz core radius of a WR star equal to twice and three times the hydrostatic core radius as well. Comparison with the observations of Guseva, Izotov \& Thuan (2000) at $Z=0.001$ shows, that a better knowledge of WR wind structures is required before conclusions can de drawn about other model parameters.

\section{Blue and red WR-bumps}

In general, binaries produce a different behaviour (because of mass transfer and merging) and cannot be neglected when studying optical spectra of WR galaxies. The scaled WR mass loss rates are required to have realistic predictions concerning the WC/WN ratio of, for instance, the SMC. Note as well that in this galaxy, the observed low WC/WN number ratio cannot be due to a metallicity scaled RSG mass loss rate, because most WR stars did not experience a RSG stage. This conclusion can be drawn from the fact that 8 of the 11 SMC WR stars have an OB companion with orbital periods on the order of $100 \mathrm{~d}$ or less (Foellmi \& Moffat 2001), meaning that they must have gone through a Roche lobe overflow stage.

As an overall conclusion, we state that theoretical simulations are able to reproduce qualitatively the spectral observations of starbursts. However, a better understanding of WR atmospheres is necessary if any firm conclusion is to be made from the blue and red WR-bumps.

A more extensive discussion can be found in Van Bever \& Vanbeveren (2002).

\section{References}

Foellmi, C., Moffat, A.F.J. 2001, in: D. Vanbeveren (ed.), The Influence of Binaries on Stellar Population Studies (Dordrecht: Kluwer), p. 157

Guseva, N.G., Izotov, Y.I.,Thuan, T.X. 2000, ApJ 531, 776

Schmutz, W., Leitherer, C., Gruenwald, R. 1992, PASP 104, 1164

Van Bever, J., Vanbeveren, D. 2002, A\&A submitted

Vanbeveren, D., van Rensbergen, W., de Loore, C. 1998, The Brightest Binaries (Dordrecht: Kluwer) 